\title{
Write accessibly
}

\author{
It's not easy.
}

$\mathrm{n}$ an editorial published last month, we wrote "Delivering suitable amounts of therapeutics to specific cells or tissues, and doing it safely and efficiently at appropriate times, is not unlike the transportation of goods - at minimum, one needs a suitable vehicle for the cargo, accurate mapping, a feasible route, an efficient last-mile strategy and proof of delivery" (Nat. Biomed. Eng. https://doi. org/10.1038/s41551-021-00798-7 (2021)).

The text is informative, clear and accessible.

How much does clarity and accessibility matter? After all, most scientific writing is read by experts. They can quickly understand lengthy scientific parlance, such as 'Many nanovehicles (NVs) for the transportation of therapeutic cargos, which can be tracked via imaging based on anatomical data, depending on the mode of $\mathrm{NV}$ administration before stimulus triggered cell- and tissue-specific controlled cargo release, are being utilized for the safe, efficient and timely NV-mediated cell delivery of targeted drugs'.

Yet even trained scientists benefit from clear and concise text that provides appropriate context. Reading well-flowing and informative text that is unambiguous, technically accurate and precise is pleasant. Taxing the reader by forcing them to 'decode' the meaning or to perform mental gymnastics to associate elements untidily spread over dependent clauses will unfavourably compete with readily available and satisfying sources of attention.
How can complex technical text be made accessible? It's not rocket science. It's not science either. And deliberate practice goes a long way. Still, we offer here a few of the lesser-known tips from the art of the trade. A caveat: there are always exceptions to the rule.

\section{Minimize the number of acronyms, and use only common acronyms}

Unlike 'NV' in the second paragraph of this article, acronyms should be used more than once to abbreviate more than one word. Using too many acronyms burdens the reader's memory and interferes with reading flow. Do not invent a new acronym when a commonly used one is available, and avoid using fancy or common words as acronyms for new methods or strategies. Uncommon gene, transcript and protein names should be defined (yet not necessarily spelled out).

\section{Convey meaningful information} precisely, and aim to use fewer words Vehicles transport cargo, and hence 'many nanovehicles for the transportation of therapeutic cargos' can be simply written as 'Nanoscale vehicles for drugs'. And 'due to the fact that the number citations to work in the field has been increasing, it's worth considering the point that' is not worth the reader's time. (And citations always increase, for all fields.) Also, stay away from qualitative modifiers for quantitative variables. Interpreting 'very large values', 'many measurements' and 'multiple images' requires appropriate context; provide numbers when available.

\section{Avoid non-standard terms and 'lazy' word amalgamation}

A 'nanovehicle' is not an accessible term for a nanoparticle for the delivery of drugs, and a supramolecular aggregate should not be shortened to 'supra-aggregate'; however, 'theranostic' and 'PEGylated' that is, 'therapeutic and diagnostic' and 'functionalized with poly(ethylene glycol)', repectively - are commonly accepted.

Beware of adjective stacking, and use word order and hyphenation to remove ambiguity and to facilitate reading flow Proper word order and punctuation aids interpretation. For example, 'NV-mediated cell delivery of targeted drugs' conveys that the drugs are targeted (rather than only the delivery vehicle, as may be the case), and the wording is unclear about what is delivering the drugs (cells, a nanoscale vehicle, or a combination of both?). And 'stimulus triggered cell- and tissue-specific controlled cargo release' requires more effort to decipher than 'stimulus-triggered cell-and-tissue-specific controlled cargo release. However, excessive punctuation is demanding of the reader. 'A stimulus triggers the controlled release of cargo to specific cells and tissues' is more considerate. Also, pay attention to the correct use of 'and' and 'or', especially when using negation.
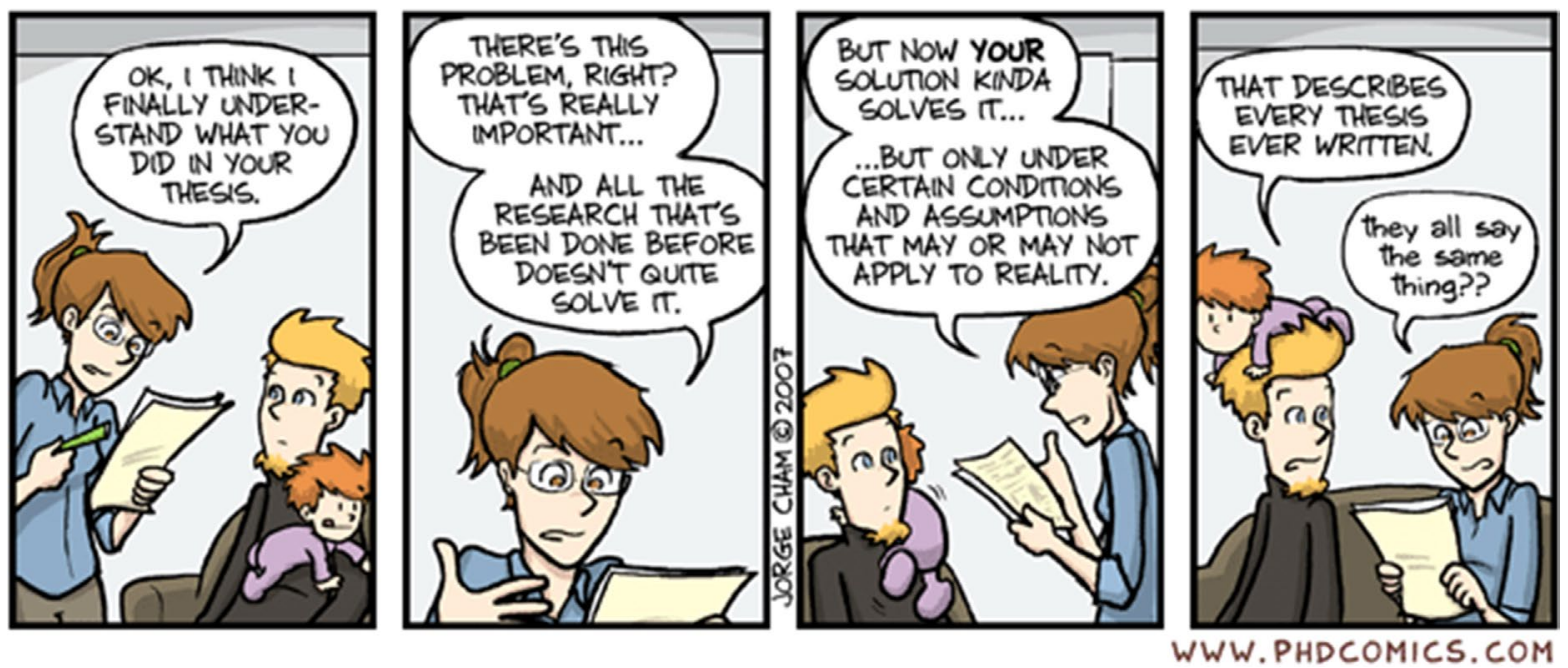

Credit: Piled Higher and Deeper by Jorge Cham. Reproduced with permission from www.phdcomics.com. 


\section{Connect every sentence and clause meaningfully with contiguous sentences and clauses}

In the sentence 'many nanovehicles (NVs) for the transportation of therapeutic cargos, which can be tracked via imaging based on anatomical data', it is unclear whether 'which' refers to the 'nanovehicles' or the cargos. And how the clause that follows, 'depending on the mode of NV administration before stimulus triggered cell- and tissue-specific controlled cargo release' relates to the rest of the text is also open to interpretation. Here is one way to remove the ambiguities: 'Nanoscale drug-delivery vehicles targeted to specific cells and tissues can be tracked via imaging techniques relying on anatomical data and the route of administration before a stimulus triggers the controlled release of the drug.

\section{The passive voice should be sparingly used}

Sentences written in passive voice don't make the work more objective and often hide the subject and the timing of the action (is a claim preceding or following 'has been shown' referring to the authors' study or to earlier work by others?). And text overusing the passive voice feels unnecessarily dry, even when describing methods and protocols.

\section{Don't repeat the same point}

Avoid repetition within the same section, even if using different wording. Readers are smart. Treat them as such. Be clear in the first instance.

\section{Refrain from biasing the reader}

Do not write that something is important or exciting. Write about the specifics of the achievement, and let readers form their own opinion about its significance.

Avoid hype (Nat. Biomed. Eng. 1, 771 (2017)). That is, abstain from wording denoting novelty, priority or importance, such as 'for the first time', 'breakthrough', 'paradigm shift', 'innovative, 'striking', 'unprecedented' and 'successfully'. Two contemporary exceptions: viruses and drugs can be novel, and there can be breakthrough infections.

\section{Emphasize appropriately}

The text quoted in the first paragraph of this article emphasizes the challenges; the unskilfully constructed sentence framed by quotation marks in the second paragraph highlights methodology, functionality and use. Ensuring suitable emphasis is particularly important for titles and abstracts, where space for context is scarce. Readers ought not to guess whether the work focuses on, for example, performance, methodology, validation or mechanistic understanding.

"Break any of these rules sooner than say anything outright barbarous", George Orwell wrote. For scientists, breaking his 'never use a long word where a short one will do' rule can be particularly pertinent. And saving words at the expense of comprehensibility is unwise. Be generous; readers won't save time if they need to re-read unclear text. When space is precious, cut content, not clarity.

Published online: 15 October 2021

https://doi.org/10.1038/s41551-021-00816-8 